\title{
XLV. On refraction
}

\section{Joseph Reade M.D.}

To cite this article: Joseph Reade M.D. (1822) XLV. On refraction, Philosophical Magazine Series 1, 59:287, 200-207, DOI: 10.1080/14786442208652719

To link to this article: http://dx.doi.org/10.1080/14786442208652719

$$
\text { 曲 Published online: } 29 \text { Jul } 2009 .
$$

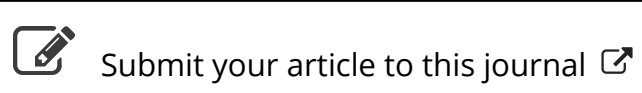

\footnotetext{
Џll Article views: 3
}

Q View related articles $\sqsubset$ 
particular time. As the scale of the barometer bears examination, and as the formula (Dr. Maskelyne's) will scareely be questioned, it is only in the specific gravity of the mercury, or in an erroneous estimate of the proportion of the area of the tube to that of the cistern $\left(\frac{1}{3}\right)$, that we can louk for the uniform discrepancies.

Comparison of the Altitudes given in the Trigonometrical Survey and in the present List (reduced to the ground).

$\begin{array}{lllrrrr} & & & \text { Table. } & \text { Trig. Survey. } & \text { Diff. } \\ \text { Ingleborough } & \ldots & \ldots & 2368 & 2361 & . . & 7 \\ \text { Pennigent } & \ldots & \ldots & 2281 & 2270 & . . & 11 \\ \text { Great Whernside } & \ldots & \ldots & 2309 & 2263 & \ldots & 46 \\ \text { Rumbles Moor } & \ldots & \ldots & 1318 & 1308 & \ldots & 10 \\ \text { Pendle } & \ldots & \ldots & 1824 & 1796 & \ldots & 28 \\ \text { Boulsworth } & \ldots & \ldots & 1692 & 1689 & . . & 3\end{array}$

The differences are in general very trivial; and may we not assign as a reason for the two marked exceptions, that the great theodolite was not used either at Pendle Hill or at Great Whernside, and that the refractions made use of in the calculations were greater than the reciprocal observations in the vicinity could warrant ?

All the angular instruments employed in these operations were made and divided by the late Mr. James Allan.

to The author is respectfully informed, should the Journal of the Thermometric Indications at the summit and base of Rumbles Moor. which he states has been kept from the beginning of February, and will be discontinued on the 1st of April, present any interesting results, that I shall be happy to make room for it in the pages of the Phil. Mag. and Journal,-A. T.

XLV. On Refraction. By Joseph Reade, M.D.

[Continued from vol. lviii. p. 254.]

$I_{N}$ my last communication I mentioned a simple, and I hope, in the opinion of men of science, a conclusive experiment against the commonly received doctrines of refraction. I shall now mention the following variation. Having procured a very clean cylindrical tumbler (fig. 7, Plate III.) with a flat bottom, about three inches diameter, I placed half-a-crown at the bottom, and holding it near a well lighted window, I poured in water by very small quantities at a time, my eye being on a plane : as soon as the object was entirely covered, a reflected image formed immediately over it, which rose with every addition of water: having 


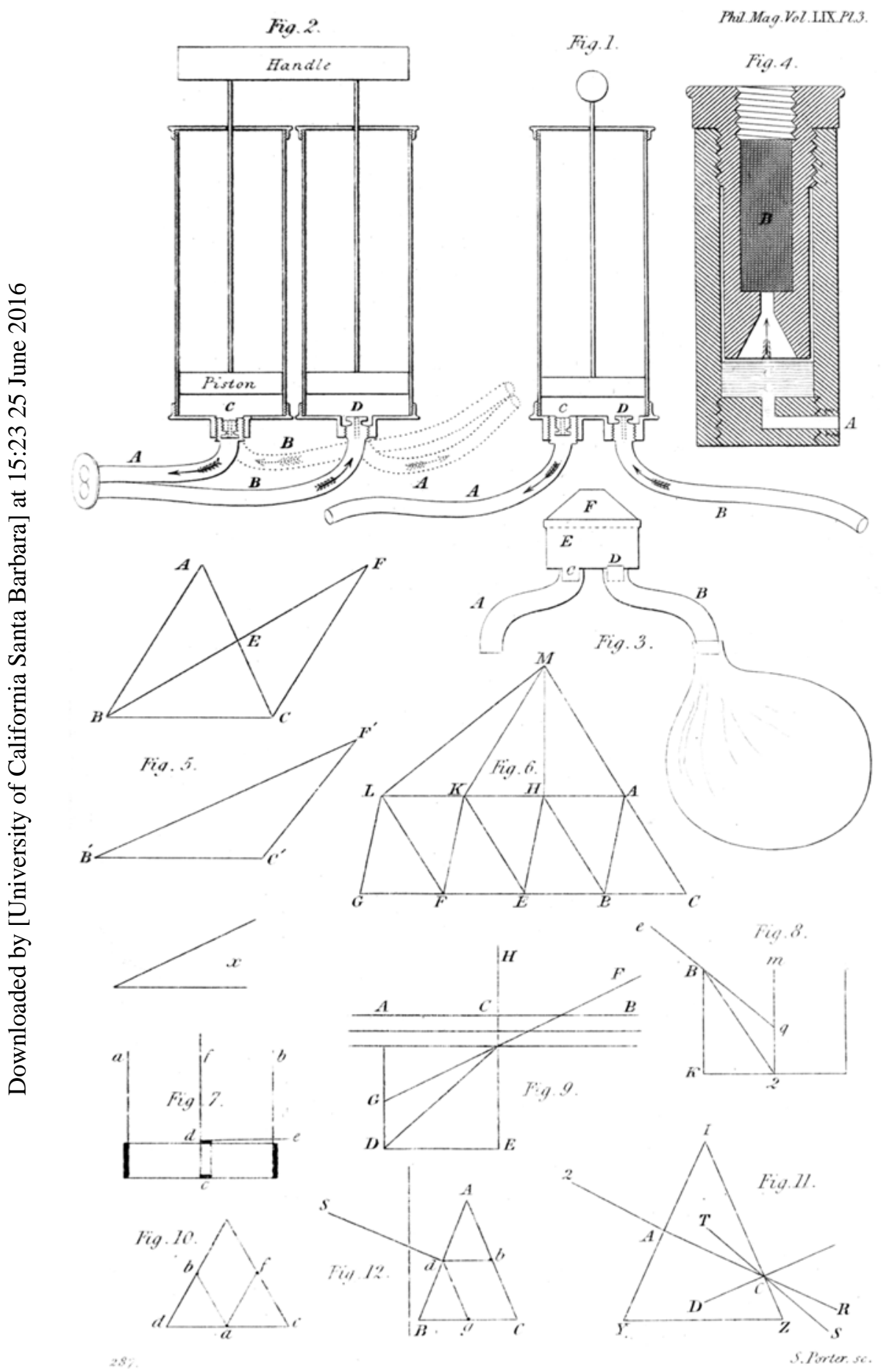


poured in to the height of about one inch, the image floated at the surface. I now covered the tumbler up to this surface with black cloth, and desired an assistant to throw in different coins, while I kept my eyes shut, each of which I described on again opening my eyes, by looking at their images floating on the surface of the water an inch above the coins, my eye being on a line with that surface, as thus represented : $a b$ a tumbler filled to the height of one inch with clear water, and covered with black cloth; $c$ a half-crown placed at the bottom; $d$ the reflected image immediately over the coin, and seen by an eye at $e$.

Now, sir, I would beg leave to ask any person, not entirely blinded by prejudice, Is there not a reflected image formed perpendicularly over the piece of money, capable of being seen by an eye above, below, and on a line with the surface? Query,-Does this reflected image send rays, or rather, an image, to the spectator's eve? To see is to believe *. But, sir, in your last Journal there is something about the analogy between reflection and refraction : however, as no particular objections are brought against my opinions, I must think it a waste of time to answer vague and angry generalities. I am well aware, that my opinions on vision, light, and colours, are diametrically opposite to those of the schools, and entertain too high a respect for their professors not to believe that they will undergo a liberal and unprejudiced examination. If the gentleman be really serious in denying the evidence of his senses, he must come to particulars.

Now let us examine this experiment according to the received laws laid down in every elementary treatise on optics; and I contend that no refraction or bending of the rays can possibly take place at $d$, for the rays $c d$ enter the air perpendicular to the plane surface of the water; consequently they must pass on without any refraction. Mr. Harris has a figure (see fig. 8.) illustrating refraction at plane surfaces. Suppose the vessel empty, BK its side, and $Q$ the object at the bottom; if the eye be at $e$, the object will be hid by the side $B K$; but by filling the vessel with water, it will become visible, and be seen at $q$. The ray $Q B$ being refracted into $\mathrm{B} e$. Mr. Harris speaks as if an image were formed in the body of the water at $q$. For the purpose of making the rays of light enter the air in an oblique direction, mathematicians have made them to diverge from the point $Q$. On the contrary, we find by direct experiment, that an image of the half-crown is formed over the piece of money, which could not be the case were the rays diverged : that it is a reflected and not

* If the rays $c d$ are refracted in the direction $e$, the rays $e$ should be refracted in the contrary direction $d c$; and an eye under the water at $c$ should perceive an object at $e$, which is inpossible; for then the sine of incidence wonld be equal to a radius.

Vol. 59. No. 287. March 1822.

$\mathrm{C} \mathrm{C}$

a re- 
a refracted image we see, is eviflent from our being able to see it in every direction floating on the surface of the water: if refracted, we could only see it in the direction of the refracted rays. When the eye is placed immediately over the half-crown, looking down into the water, we see the image, not the piece of money, one-fourth nearer to the eye : here there can be no refraction, as the rays coming to the eye must be at right angles to the surface of the water : here there is no angle of incidence; no angle of refraction; no ratio oi 3 to 4 . In fact, this simple experiment rebels against almost all the laws of optics. Snellius was the first who supposed he discovered a constant ratio in refraction; he used the secants of the complements instead of the sines used by the celebrated Des Cartes. As his doctrines are founded on this experiment, $I$ think it necessary to make a few observations.

Supposing the surface of the water to be A B (fig. 9), and an object under it at $D$, which to the eye at $F$ appeared as it were in the line $\mathrm{T} C$. He produced $\mathrm{T} \mathrm{C}$ till it met in $\mathrm{G}$ with the perpendicular $\mathrm{DA}$ to the surface $\mathrm{AB}$. Then he argued, that the image of the object $D$ appeared at $G$, and that $C D$ was to $C G$ in a certain given ratio as 4 to 3 in water.

The following objections may be made : 1. The images can be seen by an eye at $B$ on a plane with the surface of the water. 2. 'This image can be perceived in every direction above, below, and on a plane with the surface of the water, which could not be the case with a refracted image. 3 . There is no reason whatsoever that the ray $\mathrm{D} C$ should be refracted in the diagonal at plane surfaces, except for the purpose of supporting the theory. On the contrary, there is every reason to prove that the rays move parallel, for the image is perceived at $A$ immediately over the piece of coin. An eye at A looking down into the tumbler sees the piece of money one-fourth nearer. Here, according to opticians, the rays are not refiracted; yet they cannot deny that the piece of money appears nearer the eye, and somewhat magnified. If it were the object and not an image they saw, it would appear at the same distance as in air. It is agreed on all hands, that every refracting surface forms a reflected image; why resort to any other means? I shall now proceed to extend this experiment to a medium terminated by two plane surfaces inclined to one another, such as an equilateral prism.

Having placed a sovereign under the plane of a prism (fig. 10.) resting on the table, I found that two reflected and not refracted images were formed in each plane, as represented in the following figure. $a$ The sovereign placed under the plane $d c$ of an equilateral prism, forms an image at $a$; which image sends images to $b$ and $f$. That these are reflected and not refracted images, is so evitlent as scarcely to require remark. According 
to the present theory, two images could not possibly be formed by refraction at $b$ and $f$; for $a$ being at right angles to the plane $d \dot{c}$, the rays shwuld sufter no refraction, but proceed on to the vertex. Tise very same mistake which induced optical writers to suppese from analogy that rays converged in the body of a convex lens, made them also suppose that rays were turned in the body of a prism to the thickest part as well within as without the medium. Let us now examine this experiment according to the present lans of optics.

Let the angle $\mathrm{CAI}$ (fig. II) be a right angle; then the whole refraction is at $\mathrm{C}$; and in this case, $\mathrm{D} \mathrm{C} \mathrm{A}: \mathrm{A} \mathrm{C} \mathrm{D}:: m: m-n$. Also, since the right angle $\mathrm{DCI}$ is equal to the sum of the two $\triangle \mathrm{CI}$, A I C, take away the common angle $\mathrm{ACI}$, and the remaining angles DCA, A I C are equal. Consequently A I C : A C I : : m:m-n. Now I would beg leave to ask, Does any light in this experiment pass through the plane $Y Z$ ? The ray $Q A$ is undoubtedly turned to the thickest part of the prism ; not, as Newton and his followers suppose, from any principle of attraction, but simply becanse it strikes the plane I $Z$ obliquely, and there forms ain image, which moves downwards. Let us vary this experiment. I placed the plane of the prism on a sniall hole, cut in a large sheet of pasteboard, and perceived two innages of the hole formed in the planes, as alreadly described with the sovereign. I now removed this sheet of pasteboard with the prism into the sun-beams, as represented (see fig. 12), and found that the rays passed through both planes $S$. The sun passes through a hole in the pasteboard, and, striking the plane $A B$ perpendicularly, forms an image at $d$, which inage sends rays to form other images at $f$ and $g$. Here we have two speetra at $f$ and $g$, the one ascending the other descending in consequence of striking the planes obliquely. In this experiment opticians are necessarily obliged to relinquish one of their favourite law's, "that rays striking at right angles to plane surfaces suffer no refraction; for it cannot possibly be denied, lst, that the rays strike the plane $\mathrm{AB}$ at right angles; and $2 \mathrm{dly}$, that the rays diverge: otherwise they could not come through the planes $A \dot{C}$ and B C. Are the rays refracted in opposite directions? or are they attracted and repelled in opposite directions? But if we adınit that an image is formed at $d$, we can easily account for the two reflections at $f$ and $g$. Had Sir Isaac Newton been acquainted with the formation of two spectra (and I cannot but express surprise that he was not), he never could have maintained the doctrines he did. Here I rannot but notice a curious fact in regard to the prism, although not immediately connected with the doctrine of refraction. When the sun-beams are passed through the lower refracting angle, as it is called, on emergence they ascend

$$
\text { C } \mathrm{C} 2
$$

and 
and form a heautiful spectrum on the opposite wall, orange at the bottom, violet at the top, with intermediate colours : but on looking through the same refracting angle at the hole in the pasteboard or window-shutter, the experimenter is surprised to find all the colours reversed, violet at the bottom, orange at the top. Newton must have had very defective eyes, or must have been very inattentive, entirely to have overlooked this interesting fact; for we often find him in the Optics looking at the hole through the prism, yet never mentioning it. I shall explain this phenomenon in my treatise on Vision, with which it is intimately connected ; and shall merely remark, that the rays forming the spectrum have nothing to do with vision-making images. "Then," says Newton, "I looked through the prism upon the hole. In this situation, viewing through the said hole, I observed the length of its refrarted image to be many times greater than its breadth, and that the most refracted part thereof appeared violet, the least refracted red, the middle parts blue, green and yellow in order. The same thing happened when I removed the prism out of the sun's light, and looked through it upon the hole shining by the light of the clouds beyond it; and yet, if the refractions were done regularly, according to one certain proportion of the sines of incidence and refraction, as is vulgarly supposed, the refracted image ought to have appeared round." Here Newton's attention seems to bave been so completely absorbed with preconceived opinions, that he never noticed the colours being reversed; and consequently, that the image he saw on the plane of the prism and that on the opposite wall were distinct and different, bearing no analogy whatever. On looking at the hole in the window-shutter through the lower refracting angle, we are obliged to direct the optic axis on a line with the ground, and then see a reflected and not a refracted image painted on the prismatic plane.

As I have shown in the first volume of the Experimental Outlines for à new Theory of Vision, Light, and Colours, p. 48, that Newton never separated what he calls white light into seven coloured rays, I think it perfectly unnecessary to speak of their different refrangibilities : any fluid passing through a resisting medium obliquely must be lengthened; and I have shown that a straight stick, when viewed through the prism, is curved; therefore it is not surprising that the image of the hole should be ohlong, not circular, and bounded by two semicircular ends.

Here I think it necessary to mention, that when writing the Outlines I had not made the first experiment mentioned in this paper, and therefore believed in the theory of refractions. The next experiment on which the theory of refraction seems to rest, is the following: "Take an empty vessel, such as a basin, and all 
along the diameter of its bottom fix little marks at a small distance from one another; then, through a small hole in the window-shutter of a dark chamber, let in a beam of the sun's light : where the beam falls upon the floor, place your basin so that its marked diameter may point towards the window, and that the beam of light may fall on the mark that is most distant from the window: this done, fill the basin with water, and you will observe that the beam which before fell upon the most distant mark, will now, by the refractive power of the water, he turned out of its straight course, and fall two, three, or more inches nearer the centre of the basin." The fallacy of this experiment can easily be explained on the same principle as the first. I shall merely remark, that when the water is thrown in, we do not see the marks at the bottom of the basin, but reflected images of those marks floating on the water; and also the beam of light, when falling obliquely on the surface of the water, must cause a reflectea image, such as an oar would. Therefore, any conclusions drawn from such an experiment must prove erroneous.

A very simple experiment may be made in the following manner : Cut a square piece of white paper about the size of a halfcrown, and let it be dipped in a tumbler of clear water : on looking at it, it appears as if split into two papers, giving a simple but conclusive illustration of these reflected images.

I shall now say a few words on refraction through concave and convex lenses; nor do I see much occasion to enlarge on this part of my subject, having already in my paper on Vision, published in one of your former Journals, shown that the cornea and not the retina is the true and only seat of vision, and that the mind receives its ideas from minute images painted thereon, aud not from any crooked refractions forming imaginary images in the air. Indeed, a person consulting optical writers, and referring to their figures explanatory of telescopes with four lenses, must suppose Nature, instead of being simple and uniform in her operations, to be fond of all manner of twistings and turnings. At the object-glass the rays get the first twist; two more at the medium-glasses; a fourth at the eye-glass; a fifth at the cornea; a sixth at the crystalline lens; a seventh at the vitreous humour; and, if it were necessary, a dexterous optician may twist it round his finger. Newton and De Domenis have done nearly as much with their two reflections and two refractions in the rainbow.

For the experiments with lenses, it is necessary to procure a glass globe about three inches in diameter, the bull's eye of a magic-lautern, and a concavo-concave lens. Having pasted a piece of black cloth in the shape of the letter $T$ on a pane of glass at the window, I requested an assistant, when seated opposite, to look steadfastly at it: on now looking into his pupil, I per- 
ceived a beautiful reflected image of the letter T. I now placed the bull's eye immediately in front, and then perceived this image to be considerably magnified in all its dimensions and surrounded with colours : he said he saw exactly similar to the reflection on his cornea. I wonld now beg leave 10 ask, Did this gentleman perceive the letter $\mathrm{T}$ by a reflected or refracted image? On removing the bull's eye to yet a greater distance from the pupil, I distinctly perceived two reflected images, the one erect, the other inverted. Again I would ask, Is it possible by refraction to produce in the focus of a lens both an erect and an inverted image at one and the same time? That we see by means of reflected and not refracted images, is therefore evident. This experiment is easily repeated with the glass globe instead of an assistant's eye. On a sheet of white paper write the letter T, and hold over it the bull's eye : when close to the paper, the letter is considerably magnifed : on bringing it somewhat nearer to the eye, two inverted and coloured images are perceived to float on the posterior surface. On now giving a circular motion to the lens, these reflected images, in revolving round the erect one, become inverted or erect; when at the top and bottom they are inverted; when at the sides erect; for which phænomenon I am as yet unable to account. At yet a greater dtstance these two images form a circular appearance, margined on the inside by orange rays, and at length coalescing form one inverted image, which floats around the erect image with each revolution, without charge. When we look at an object, its picture is painted on the cormea, and thence converges to the sensorium in the same manner as with the other senses. By placing a concave lens before the eye, this reflected image is diminished; by placing a convex one it is magnified. A short-sighted person sees objects large and confused when at a distance; a concave lens obviates this defect, by painting a small and well-defined image close to the eyes; for a near-sighted person can read small print when near without glasses. In old age the humours become decayed and turbid, and the corneal image is not sufficiently strong to make an impression on the retina, the principal nerve of the eye. Therefore a convex lens is necessary for the purpose of forming a magnified image closer to the eye, and also for the purpose of illuminating that image and throwing a greater quantity of light into the eye. Any person may make himself near-sighted either by coustantly examining near and small objects, or by the wearIng concare glasses; for by these means the eye becomes accustomed to the strong stimulus of rays from near objects, or from the images near the eyes. In a similar manner, a person may make himself deaf, by constantly accustoming the ear to intense noises, such as the roar of camson, \&c. 
Mr. Ware has written an excellent paper on the use and abuse of glasses. Perhaps it may be objected to the first experiment of this paper, that the piece of money radiated light as if from a centre or focus. To obviate which, I varied the experiment in the following manner: I first placed the piece of money at the bottom of the tumbler, and then placed immediately on it a concavo-concave lens; on filling in the water, I found the image formed, as already represented. I now placed a plano-convex lons over it, with the same results : here the rays were reflected to a focus, and consequently they could not answer for a refracted image.

The theory of refraction and the retinal theory of vision are so intimately and inseparably united, that the one cannot exist without the other. I therefore would request Mr. Stark to read my paper on Vision, published in a former Journal. If I have expressed myself with too much confidence, I must express my regret, and hope the learned and candid reader (for learning and candour generally go hand in hand) may attribute it to haste, perhaps not unaccompanied by a feeling of resentment at prejudice and critical neglect. But, sir, I am now happy to see that my opinions are daily gaining ground, and sanctioned by men of the first-rate abilities. I am certain both $\mathrm{Mr}$. Stark and myself have one and the same object in view, the discovery of truth. I therefore shall endeavour, as far as hes in my power, to answer any particular objections, but must decline a metaphysical controversy on the nature of light; especially as the theory of Newton or that of Des Cartes would equaily answer for experimental inquiry. Disputatio torquet homines, says Cicero; and impressed with a high respect for that great orator, I would wish to avoid it.

Epicurus thought that vision was produced by a continual suecession of material images sent to the eye, which at their first emission from the object are large, decreasing continually the further they go, till they arrive at such a smallness as will permit them to enter the eye. That inages are sent off from bodies, can easily be shown. And if I bave shown that the rays of light coming from all points of an object, and meeting again at the focus, do not make a picture of the object on any white body interposed, then we have no other alternative than to go back to Democritus and Lucretius.
I remain, sir, your obelient servant, Cork, Feb. 26, 1822. JUSEy'h ReADE, M.D. 